\title{
Preparation of megabase DNA from adult insects and mammalian spleen for pulsed-field gel electrophoresis
}

\author{
VANI BRAHMACHARI*1, R. NAGASUMA ${ }^{2}$ and \\ SAMIR K. BRAHMACHARI ${ }^{2}$ \\ ${ }^{1}$ Department of Microbiology and Cell Biology, and ${ }^{2}$ Molecular Biophysics Unit, Indian \\ Institute of Science, Bangalore 560 012, India
}

MS received 18 September 1989

\begin{abstract}
While standard techniques for obtaining megabase-size DNA from microorganisms and cells in tissue culture are now available, new methods are needed for handling solid tissues and, in the case of small-sized animals, whole organisms. Here we describe a simple and rapid method for preparing large DNA molecules from mammalian spleen, whole insects of Drosophila and Planococcus lilacinus, a mealybug. Briefly the method involves gentle steps for preparing cell suspensions and handling of these cells within agarosc blocks for preparing the DNA. In mammals like mice, the spleen, rather than the liver, is the organ of choice because, in the latter, endogenous nuclease activity is high.
\end{abstract}

Keywords. Pulsed-field gel electrophoresis; solid tissue; mcgabase DNA; Drosophila; mealybug.

\section{Introduction}

The development of techniques such as pulsed field gel electrophoresis (PFGE) has dramatically changed the strategies for dealing with chromosomal DNA because they allow manipulation of molecules in the megabase $\left(1 \times 10^{6}\right.$ base pair $)$ size range (Schwartz et al. 1983). Hand in hand with PFGE, methods for the preparation of DNA samples with minimal damage to the DNA are also being developed. Techniques for preparation within agarose blocks (inserts) of "intact" DNA from E. coli, fungi, protozoa and mammalian cells in culture are now available (Smith et al. 1988). However, standard methods for preparing DNA suitable for PFGE from solid tissues are not yet available. DNA from agarose blocks made with isolated nuclei show extensive degradation on PFGE, probably due to activation of endogenous nucleases. We report here a rapid and simple method for obtaining megabase size DNA from solid tissues such as those from organs like the mammalian spleen and from whole adult insects of Drosophila and a homopteran insect, Planococcus lilacinus (a mealybug).

\section{Materials and methods}

Six- to eight-week old Balb/c mice were used for spleenectomy. Whole insects of Drosophila melanogaster and a mealybug Planococcus lilacinus were used to prepare DNA for PFGE. Phosphate buffered saline containing 0.25 M EDTA (buffer A) was

\footnotetext{
"Author for communication
} 
used for the preparation of cell suspension (see below). Buffer containing $0.5 \mathrm{M}$ EDTA, $1 \%$ lauryl sarcosine and $2 \mathrm{mg} / \mathrm{ml}$ proteinase $\mathrm{K}$ (ESP) was used for digestion of cells within the agarosc blocks (Smith et al. 1988). Modified $1 \times \mathrm{T} 3 \mathrm{E}$ which contains $0 \cdot 1 \mathrm{M}$ Tris base, $0 \cdot 1 \mathrm{M}$ boric acid and $1.0 \mathrm{mM}$ EDTA was used in PFG electrophoresis (Smith et al. 1988).

$\lambda$ concatamers were prepared from lambda phage according to the proccclure of Greg et al. (1987). Yeast (S. cerevisiae) inserts were prepared according to Sniith et al. (1988).

\section{Results and discussion}

Inserts prepared from isolated nuclei show extensive degradation of DNA (Smith et al. 1988). Intact DNA molecules are therefore most conveniently obtained from cell suspensions. Cell suspensions were prepared from different organs like tlie liver or the spleen by squashing tlic tissue over an autoclaved metal mesh (No. 120, $0.125 \mathrm{inm}$ Endecolts filter Ltd., England). The crude suspension of cells obtained was taken up in buffer $\mathbf{A}$ and washed twice with the same buffer. $\mathbf{A}$ brief hypotonic shock was given to remove the RBC. Tlie cells were washed in buffer $\mathbf{A}$ twice and suspended at a density of $2 \times 10^{7}$ cells/ml in buffer A. Agarose inserts were prepared by adding an equal volume of $1.5 \%$ agarose, and $100 \mu \mathrm{l}$ inserts were made in tlie LKB insert mould (Smith and Cantor 1987). Inserts were then digested for two days in ESP. Pulsed field gel electrophoresis was carried out in an LKB Pulsaphor, at $15^{\circ} \mathrm{C}$ for $30 \mathrm{~h}$ at $4 \mathrm{~V} / \mathrm{cm}$ with $600 \mathrm{~s}$ pulse time followed by $42 \mathrm{~h}$ at $6 \mathrm{~V} / \mathrm{cm}$ with $100 \mathrm{~s}$ pulse time. Cell suspensions were prepared from Drosophila and a mealybug Planococcus lilacinus by the same procedure. Wings were removed before processing the flies. Mealybugs were given brief washes, twice with cold acetone to remove the waxy material covering the bugs. The separation of DNA molecules on PFGE is shown in figure 1. Whereas megabase-size DNA was obtained from spleen and whole insects, DNA samples prepared from liver tissues showed extensive degradation, probably due to the presence of endogenous nuclease activity (figure 1). The average size range of the DNA so obtained is greater than one megabase pair (figure 1). The pulse time (600s and $100 \mathrm{~s})$ used in these experiments does not favour the separation of DNA molecules larger than one megabase pair. The estimated DNA content of the haploid genome of mealybug is $0115 \mathrm{pg}$ or $1 \cdot 1 \times 10^{8}$ basepairs (K. Muralidharan, unpublished). There are five chromosomes in haploid nuclei, and on metaphase plates of diploid cells all five pairs appear almost equal in size (Brown and Cliandra 1977). On the basis of these two criteria, the size of each mealybug chromosome is estimated to be roughly 20-22 megabase pairs. Rut following PFG, tlie mealybug genome separates into three zones. A detailed analysis of tlie DNA of mealybug chromosomes would be of considerable interest because of certain nearly unique characteristics of this genetic system. Among them is the property that in male insects 5 paternal chromosomes are selectively inactivated; whereas all the ten chromosomes (5 paternal and 5 maternal) are active or potentially active in female mealybugs (Brown and Chandra 1977).

In figure $l a$, the DNA in the lane marked 'Sxr' was prepared from a cell line derived from a sex-reversed XX male mouse (Vani Brahmachari and H. S. Chandra, unpublished results). The DNA prcparcd from mouse spleen is of as high a molecular weight as that from the cell line. The method described here thus appears 

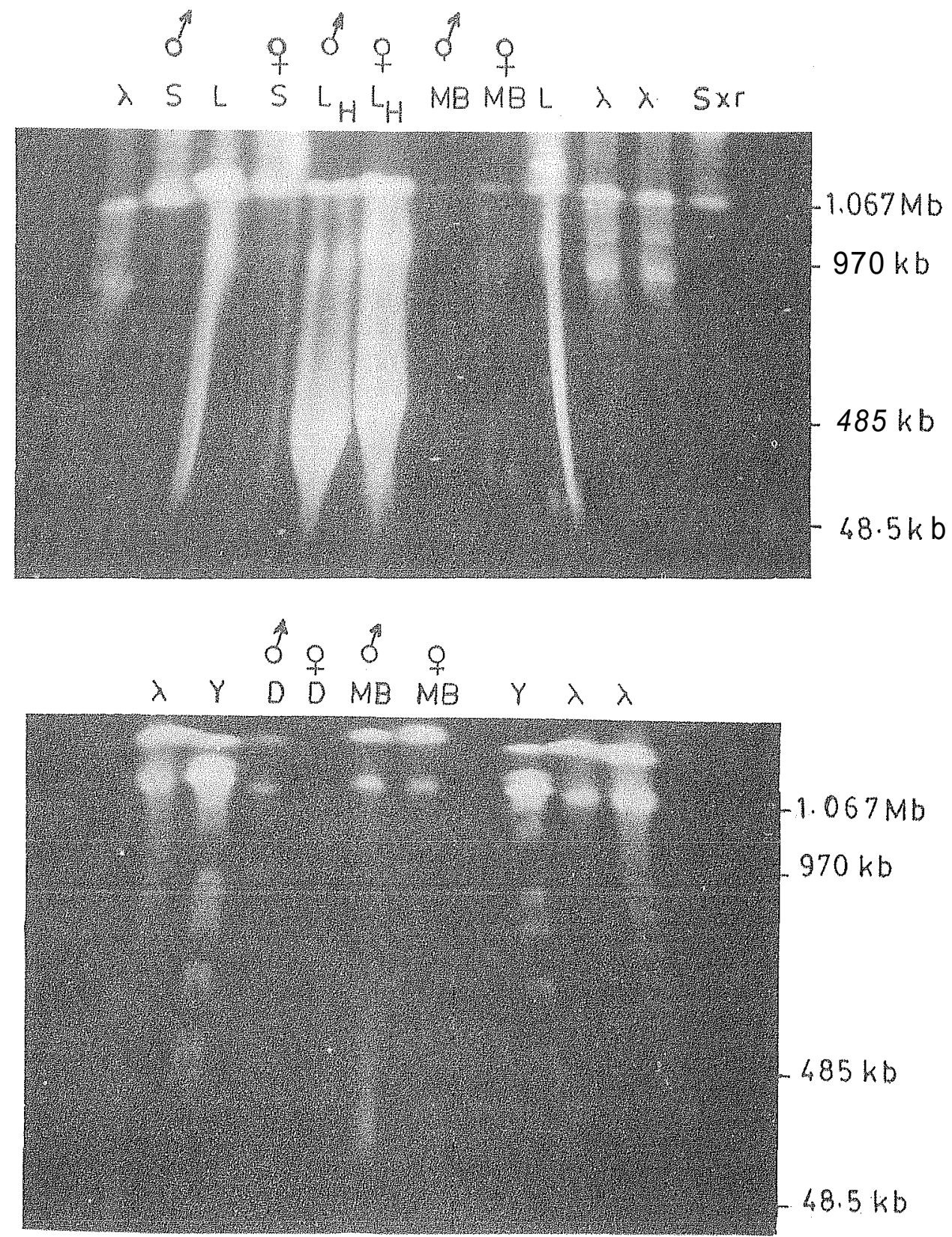

Figure 1. Pulsed field gel electrophoresis of chromosomal DNA on 1\% agarose (Bio-Rad) gel. Lambda phage concatamers $(\lambda)$ and S. cerevisiae $(Y$ thromosomes used as size markers. Mouse DNA from: spleen ( $\mathrm{S}_{\hat{O}} \hat{\mathrm{C}}$ and $\mathrm{SO}$ ), liver (L), and liver homogenized ( $\mathrm{L}_{\boldsymbol{H}} \hat{\delta}$ and $\mathrm{L}_{11}$ ); mealybug DNA (MB $\hat{f}$ and MBg) and Drosophila DNA (Dof and Dof).

to yield chromosome-sized DNA molecules from solid tissues. The usefulness of this method of insert preparation is that well-studied genetic systems like mice and Drosophila can be characterized and it may be possible to correlate specific 
phenotypic mutations with lesions at the level of chromosomal DNA. In large mammals, lymphocytes from blood samples often serve as a convenient source of DNA, but it is not practical to obtain sufficient quantities of blood from small mammals like mice, and subsequently keep the animals for further breeding or for other analyses. However, by partial spleenectomy it is possible to obtain sufficient cells for insert preparation. Recently a method for handling frozen mammalian tissues other than from the spleen has been described (Petrukin et al. 1988), where the largest fragment of known size detected is $0.6 \mathrm{Mb}$. By the method described here, mouse spleen yielded DNA of sizes greater than $1.0 \mathrm{Mb}$. On the other hand, when liver tissue was used, apparently because of endogenous nuclease activity, extensive degradation was observed in the DNA although the same procedure was employed. Thus there can be differences in the quality of DNA prepared from different organs of the same organism and the spleen appears to be an organ of choice for mammalian DNA for PFGE.

Thus the method described here appears to have features suitable for systems where established cell lines are not available. In addition, in systems like D. melanogaster, application of this method to the analysis of DNA from flies carrying known chromosomal deletions may permit correlation of phenotypic changes with DNA level changes.

\section{Acknowledgement}

We thank Prof. H. Sharat Chandra for valuable suggestions and encouragement. Financial support from the Indian Council of Medical Research through a grant to Prof. Sharat Chandra, and from the Department of Science and Technology (ILT programme) to Dr. S. K. Brahmachari are acknowledged.

\section{References:}

Brown S. W. and Chandra H. S. 1977 Chromosome imprinting and the differential regulation of homologous chromosomes. In Cell biology: $\boldsymbol{A}$ comprehensive treatise (eds) S. Goldstein and D. Prescott (New York: Academic Press) pp. 109-187

Greg P,, Waterbury and Jane M. J. 1987 Preparation of bacteriophage concatamers. Nitcleic Acids Res. 15: 3930

Petrukhin K. E., Broude N. E. and Smith C. L. 1988 A simple and efficient method for isolating high molecular weight DNA from frozen mammalian tissue. Nucleic Acid Res. 16: 5698

Smith C. L. and Cantor C. R. 1987 Purification, specific fragmentation and separation of large DNA molecules. In Methods in enzymology (eds) R. Wu (New York: Academic Press) pp. 449-467

Smith C. L., Klco S. and Cantor C. R. 1988 Pulsed field gel electrophoresis and the technology of large DNA molecules. In Genome analysis: A practical approach (eds) K. Davies (Oxford: IRL Press) pp. 41-72

Schwartz D., Saffan W., Welsh J. and Hass R. 1983 New techniques for purifying large DNAs and studying their properties and packaging. Cold Spring Harbor Symp. Quant. Biol. 47: 189-195 